\title{
High frequency separation of suspended micro/nanoparticles
}

\author{
V. Ostasevicius*, E. Golinka*, V. Jurenas*, R. Gaidys** \\ *Institute of Mechatronics, Kaunas University of Technology, Kaunas, LT - 44244, Lithuania, \\ E-mail:vytautas.ostasevicius@ktu.lt; evgenija-golinka@rambler.ru; vytautas.jurėnas@ktu.lt \\ **Department of Mechanical Engineering, Kaunas University of Technology, Kaunas, LT - 44244, Lithuania, \\ E-mail: rimvydas.gaidys@ktu.lt
}

cross $^{\text {ref }}$ http://dx.doi.org/10.5755/j01.mech.23.3.17980

\section{Introduction}

Separation of micro / nanoparticles is of great importance in chemical and biological analysis, food and chemical processing and environmental assessment. The use of high-frequency ultrasound in liquid separation systems has been knew for a long time and is due to a number of factors. First of all, it is the ability of ultrasound radiation power to capture and distribute particles of an organic or inorganic suspension along the nodes and anti-nodes. Using unique characteristics of microscale flow phenomena, various methods have been developed for the rapid and accurate separation and sorting of microparticles in a continuous mode. Advances in microfluids enable the use of sorting technologies that combine the advantages of continuous operation with small-sized scale suitable for manipulating and probing individual particles or cells. Microfluid sorting platforms require a smaller sample volume, which has several advantages in terms of reagent cost reduction, analysis time and less invasiveness of patients for sample extraction.

In the literature, methods of passive and active separation and sorting are reported. Passive methods use the interaction between particles, the flow field and the channel structure and do not require external fields. Active methods use external fields in various forms, but offer better performance. Extensive investigation of various passive and active methods of separation is presented in [1], including the basic theory and experimental data. The principles of operation are explained, and the characteristics of the devices are discussed.

The ability to obtain well defined chemical ideal microenvironment conditions and to control chemical and thermal changes, provides high resolution cell response and the interaction between cells opportunity [2]. This is the main motivation for the rapid growth of research on cell biology based on laboratory studies.

In work [3], methods currently available for selection by shape and size of nanoparticles are considered. The discussed methods vary from known chromatography, centrifugation or filtration methods to purification schemes based on phenomena characteristic of nanoscale, including selectivity in shape, or the tendency to form organized superstructures.

Recent nanostructures research achievements led to new synthetic methods that provide microparticles size, morphology and nano / microstructures be controlled [4]. The high-intensity ultrasound offers a simple, versatile tool for nanostructures, which are often inaccessible to traditional methods. The main physical phenomena associated with ultrasound, which is related to materials synthesis, are cavitation and sputtering.

Manipulation with particles in microfluidics is a problem that continues to affect applications, from the production of fine chemicals and ending with materials and life sciences [5]. Heterogeneous operations performed in micro reactors include high surface-to-volume characteristics that minimize heat and mass transfer resistance, providing precise control of reaction conditions. Significant advances have been made in the development of technologies that control particles in a microscale laminar flow, but there remain enormous opportunities for improvement in the field of chemical processing. Molecular methods change our understanding of cell function and disease [6]. However, accurate methods for molecular analysis are limited if the input DNA, RNA or protein is not derived from pure cell populations, or contaminated with erroneous cells. A new method for ultrasonic vibrations microdissection was to offer a pure population of target cells from tissue sections for subsequent analysis. A multilayer piezoelectric actuator is used to drive a vibrating needle at low amplitude and high frequency and to cut the tissue.

Innovative holographic imaging technology used to characterize the nonlinear dynamics of the vibrating structures [7, 8]. The phenomenological model of the nonlinear Duffing is appropriate to study the nonlinear response of the piezoelectric transducer, which actuates liquid with micro/nanoparticles. The method of contactless holographic characterization is suitable for obtaining results comparable to the phenomenological model.

The novelty of this work is to propose the method of micro/nanoparticles separation from fluids using disc form piezo actuator. The proposed method ensures the rapid and without damage separation process, which could be used for the cell membranes of biological fluids. Numerical simulation and holographic experimental techniques have revealed the peculiarities of disc form piezo actuator vibrations ensuring the most suitable micro/nanoparticles separation process efficiency.

\section{Piezo transducer bimorph dynamics simulation}

For acoustic waves excitation the circular piezo transducer bimorph composed from piezo-element and bronze disk was chosen (Fig. 1).

The vibration modes of piezo transducer bimorph fixed in all perimeter were calculated using the COMSOL Multiphysics software package (Fig. 2). These modes are similar to those of the circular membrane. The first (a) at 
eigenfrequency $4486 \mathrm{~Hz}$, the second (b) and the third (c) at $9389 \mathrm{~Hz}$ and $13855 \mathrm{~Hz}$ accordingly.

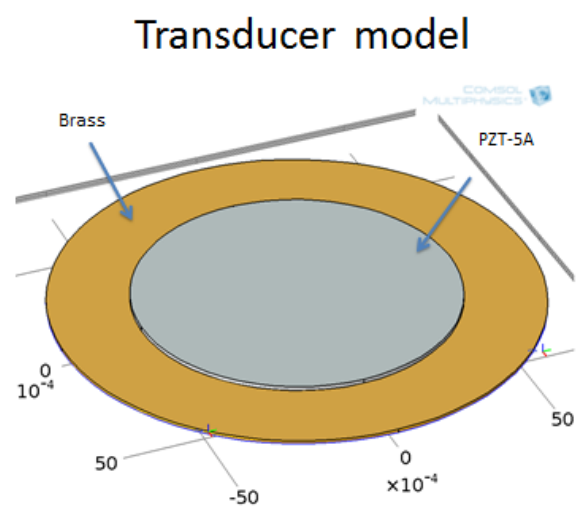

a

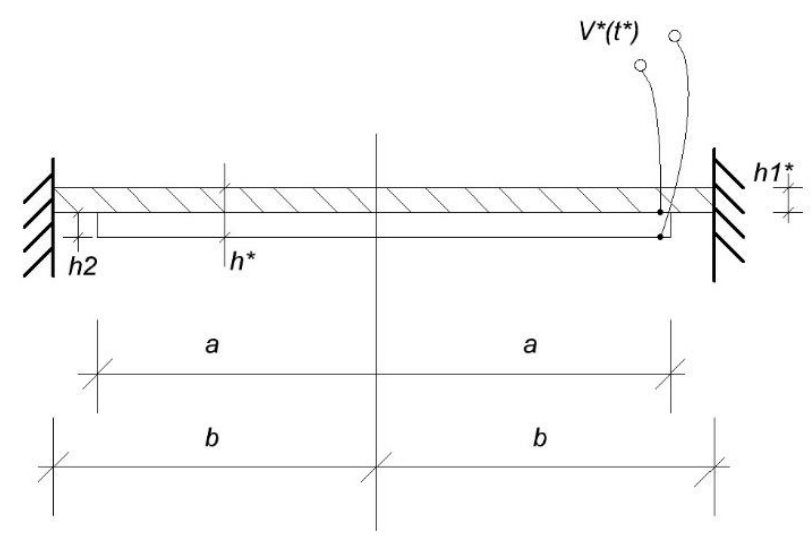

b

Fig. 1 Scheme (a) and computational scheme (b) of circular piezo transducer bimorph

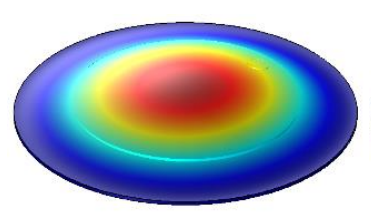

a

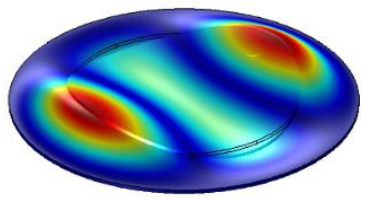

b

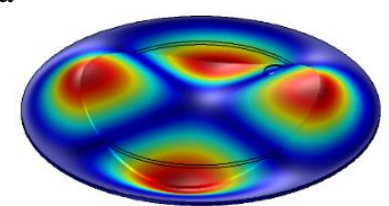

Fig. 2 Modes of vibration of transducer fixed along perimeter: a - I mode $4486 \mathrm{~Hz}$; b - II mode at $9389 \mathrm{~Hz}$ and c - III mode at $13855 \mathrm{~Hz}$

Among all possible types of free elastic vibrations it is possible to identify modes of oscillations whose frequency spectrum corresponds well to the experimental results of micro/nanoparticles separation theoretical calculations in the approximation of plane motion. Such modes of oscillations are capable to excite acoustic standing waves in fluids under consideration.

Standard acoustic problems are related to the acoustic pressure $p$ variations at the top of the stationary background pressure $p_{0}$. Mathematically this means a small parameter expansion around the stationary quiescent values. The governing equations for compressible lossless (without thermal conductivity and without viscosity) fluid flow problem are the momentum (Euler's) and continuity equations. It is calculated by the following formula:

$$
\begin{aligned}
& \frac{\partial u}{\partial t}+(u \nabla) u=-\frac{1}{\rho} \nabla p ; \\
& \frac{\partial \rho}{\partial t}+\nabla(\rho u)=0,
\end{aligned}
$$

where $\rho$ is the total density, $p$ is the total pressure, and $u$ is the velocity.

In classical theory all thermodynamic processes are reversible and adiabatic, known as an isentropic processes. The small parameter expansion is carried out with a fixed density $\rho_{0}$ of the fluid (SI unit: $\mathrm{kg} / \mathrm{m}^{3}$ ) and pressure $p_{0}$ (SI unit: $\mathrm{Pa}$ ) such that:

$$
\begin{aligned}
& p=p_{0}+p^{\prime}, \\
& \rho=\rho_{0}+\rho^{\prime}, \text { with } \quad p^{\prime} \ll p_{0}, \\
& u=0+u^{\prime}, \quad \rho^{\prime} \ll \rho_{0},
\end{aligned}
$$

where the primed variables represent the small acoustic variations.

Inserting these into the governing equations and only retaining terms linear in the primed variables yields:

$$
\begin{aligned}
& \frac{\partial u^{\prime}}{\partial t}=-\frac{1}{\rho_{0}} \nabla p^{\prime} \\
& \frac{\partial \rho^{\prime}}{\partial t}+\rho_{0}\left(\nabla u^{\prime}\right)=0 .
\end{aligned}
$$

The design of device consists of a piezoelectric actuator enclosed within a block [10]. The pressure is applied to generate the required output that is the voltage. The equations involved in the calculation:

$$
V=g t_{b} \text { stress, }
$$

where $V$ is output voltage, $g$ is voltage constant, $t_{b}$ is thickness of the actuator base block (radius $=35 \mathrm{~mm}$, height $=$ $=2 \mathrm{~mm}$ ).

Stress applied to the piezoelectric element could be found by the equation:

$$
\text { stress }=\text { E strain, }
$$

where Young's modulus $E=62 \mathrm{GPa}$.

The strain on the piezo transducer bimorph is:

$$
\text { strain }=(\text { change in piezo length }) /(\text { original length }) \text {. }
$$

After carrying out the modeling of a water-column and piezo transducer bimorph, the following results were obtained. Oscillations in the first mode were at $1.4 \mathrm{kHz}$ (Fig.3, a), the second mode was $9.5 \mathrm{kHz}$ (Fig.3, b), the third at $13 \mathrm{kHz}$ (Fig.3, c). The simulation results are presented from two positions - the type of deformation from the side of piezo ceramics in the upper line (I) and from the side of the liquid column surface in the lower line (II) of Fig. 3. 


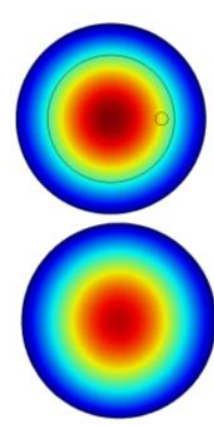

a

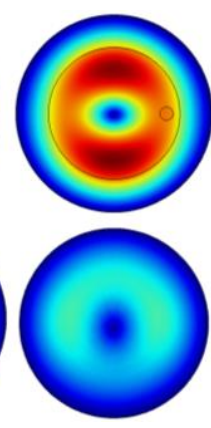

b

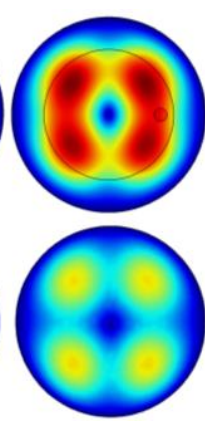

c
Fig. 3 Results of a piezo transducer bimorph modeling in interaction with a liquid column. I - line, which shows the oscillations modes from the side of piezoceramics; II - line shows the oscillations modes from the side of viscoelastic fluid: a - first mode at $1.4 \mathrm{kHz}, \mathrm{b}$ - second mode at $9.5 \mathrm{kHz}$ and $\mathrm{c}$ - third mode at $13 \mathrm{kHz}$

Based on the results of the simulation, it is seen how the oscillations of the piezo transducer bimorph are changed upon contact with the fluid. The value of the deformations decreases, and after passing through the fluid, it significantly weakens. Another interesting fact is that the force of pressure drops when the resonance mode increases.

\section{Experimental validation of the modeling results}

To study the proposed theory, a piezo transducer bimorph with a range of operation from $1.3 \mathrm{kHz}$ to $25 \mathrm{kHz}$ was used. For the validation of simulation results the holographic interferometry setup was used.

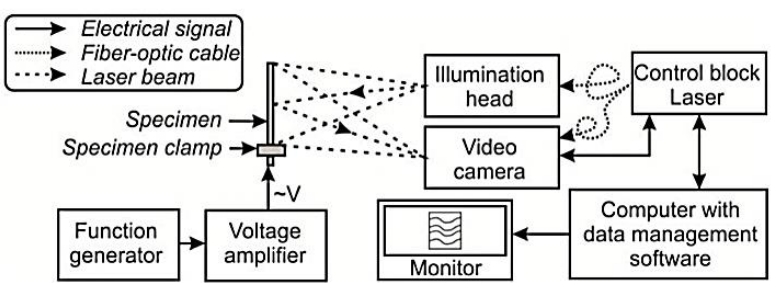

Fig. 4 Scheme of the experimental set up with laser holographic interferometry system

Fig. 4 shows the scheme of the experimental set up for recording holographic interferograms of a vibrating piezoelectric bimorph and Fig. 5 shows photo of experimental setup with holographic system PRISM 100 (Hytec, Los Alamos, NM, USA). PRISM system was used to evaluate the electrical excitation response of the investigated specimen piezoelectric bimorph. This method allows measuring vibration and deformation with minimal specimen preparation and with no contact with the specimen surface.

In the experiments, micro particle suspensions of water were used. As a micro particle phase in the suspension, the material of zeolite with color pigments was used [11]. Micro particle dimensions were 0.5-15 $\mu \mathrm{m}$.

To determine the working and resonant frequencies of the piezo transducer bimorph, a study of a loaded actuator on a holographic installation was initially carried out. The results showed that the first mode of the piezo transducer bimorph is $1.3 \mathrm{kHz}$ at a voltage of $200 \mathrm{mV}$, the second is $11.9 \mathrm{kHz}$, and third is $13.4 \mathrm{kHz}$ (Fig. 6).

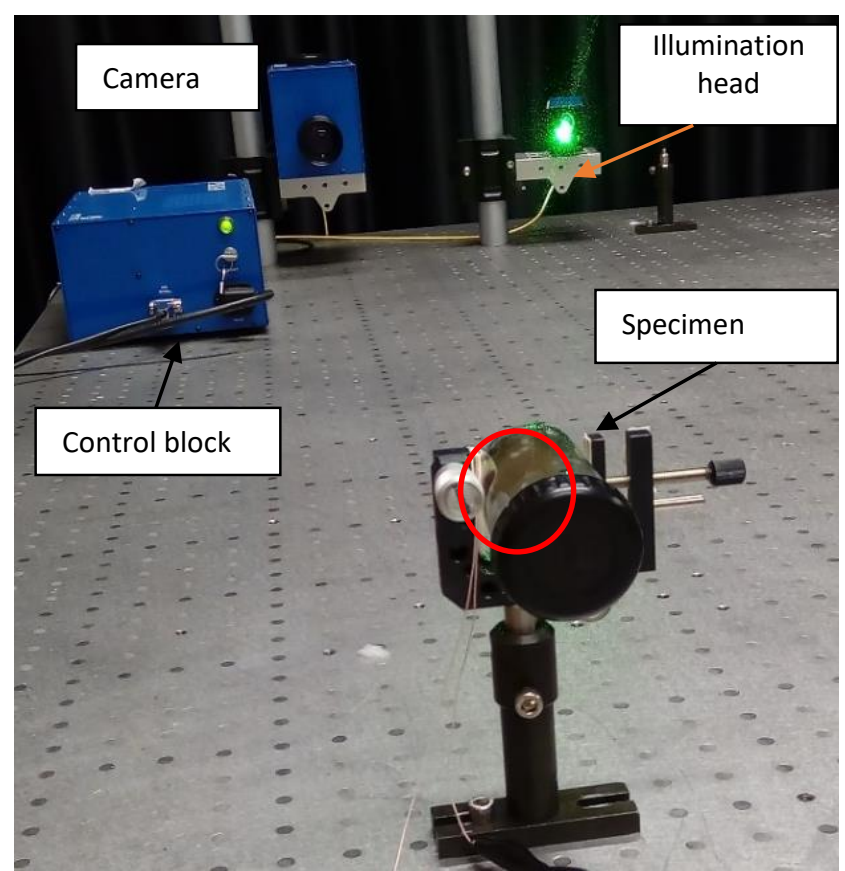

Fig. 5 Photo of the holographic interferometry setup used in the experiment
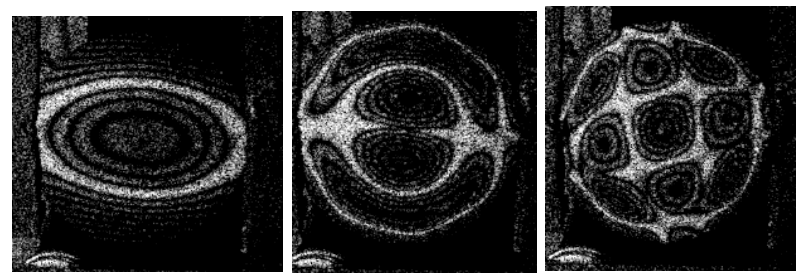

Fig. 6 Holographic pattern of the change in the surface of piezo transducer bimorph, depending on the resonance mode

\section{Micro/nanoparticles separation results}

Available resources and competencies related to the possibility of micro/nanoparticles separation from fluids have required proclaiming the sequence and results of this procedure to ensure the simplicity and quick usage of the separation process. Starting from the choice of COMSOL Multiphysics software for simulation, which imitates all real conditions of the liquid systems, the choice of a disc form piezo transducer bimorph was successful. The original experimental setup was assembled in order to validate the simulation results.

The experimental setup is presented in Fig. 7, a. During the experiment, it was found that after 5-8 seconds from the excitation signal applied to the resonator micro / nanoparticles begin to accumulate around the acoustic nodal points thus stratifying in the test container sections disposed at same distances from each other. The third and most stable resonance result was at $12.2 \mathrm{kHz}$. For the clarity of the experiment, observations were made regarding the effect of the amount of fluid in the test container on the result. As a result, a proportional number of concentrated clusters of particles fractions of the suspension was obtained, depending on the height of the liquid column (Fig.7, b and c). 


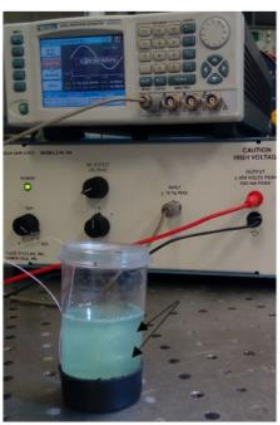

a

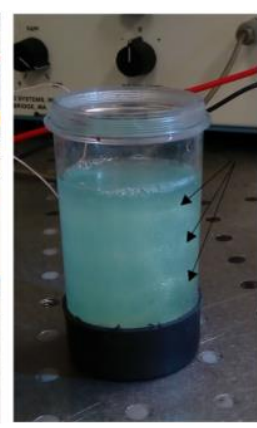

b

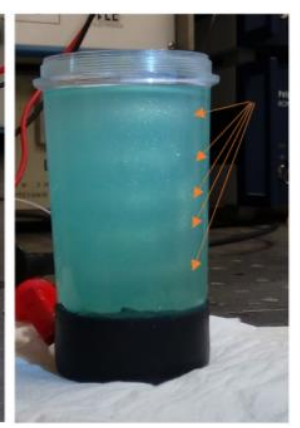

c
Fig. 7 Experimental setup (a) and micro/nanoparticle concentration rings $(b, c)$, depending on the height of the fluid column at a frequency of $12.2 \mathrm{kHz}$

\section{Conclusions}

A method and conceptual device to continuously separate micro/nanoparticles in a suspension is reported. Acoustic forces in a standing wave field are utilized to discriminate particles in suspension. Numerical simulation results confirmed by an experimental test indicate that the proposed purification procedure is feasible. A mathematical model of the suspension excitation has allowed us to determine the low-pressure areas of acoustic waves, which collect micro particles. The obtained results are a prerequisite for the development of an experimental prototype for micro/nanoparticles purification.

\section{Acknowledgments}

This work has been funded by a grant (No. SEN-10/15) from the Research Council of Lithuania. Project acronym: "CaSpine".

\section{References}

1. Sajeesh, P.; Sen, A.K. 2014. Particle Separation and Sorting in Microfluidic Devices: a Review. Microfluidics and Nanofluidics Vol. 17(1), 1-52. http://dx.doi.org/10.1007/s10404-013-1291-9.

2. Nilsson J.; Evander M.; Hammarström B.; Laurell T. 2009. Review of Cell and Particle Trapping in Microfluidic Systems. Analytica Chimica Acta 649 (2009), 141-157. http://dx.doi.org/10.1016/j.aca.2009.07.017.

3. Kowalczyk B.; Lagzi I.; Grzybowski B. 2011. Nanoseparations: Strategies for Size and/or Shape-selective Purification of Nanoparticles. Current Opinion in Colloid \& Interface Science 16, 135-148. http://dx.doi.org/10.1016/j.cocis.2011.01.004.

4. Jin Ho Bang; Suslick K. 2010. Applications of Ultrasound to the Synthesis of Nanostructured Materials. Adv. Mater. Vol. 22, 1039-1059. http://dx.doi.org/10.1002/adma.200904093.

5. Flowers B.; Hartman R. 2012. Particle Handling Techniques in Microchemical Processes. Challenges Vol. 3, 194-211. http://dx.doi.org/10.3390/challe3020194.

6. Chen1 L.G; Sun1 L.N.; Liu1 Y.X.; Wang H.X. 2007. A Novel Micro-dissection Method Using Ultrasonic Vibration for Molecular Analysis. Engineering Materials Online: Vol. 339, 291-296.
7. Ostasevicius, V.; Ragulskis, M.; Palevicius, A.; Kravcenkiene, V.; Janusas, G. 2005. Applicability of Holographic Technique for Analysis of Non-linear Dynamics of MEMS Switch. Smart Structures and Materials 2005: Smart Electronics, Mems, BioMems, and Nanotechnology, Book Series: Proceedings of SPIE, 5763: 405-413. http://dx.doi.org/10.1117/12.599027.

8. Ostasevicius, V.; Palevicius, A.; Daugela, A.; Ragulskis, M.; Palevicius, R. 2004. Holographic Imaging Technique for Characterization of MEMS Switch Dynamics. Int. conf. Smart Structures and Materials 2004.

9. Kang, M-G; Jung, W-S; Kang, C-Y; Yoon, S-J. 2016. Recent Progress on PZT Based Piezoelectric Energy Harvesting Technologies Actuators Vol. 5(1), 5.

10. Deshpande, A.; Naduvinamani, S. 2012. Energy Generation Using MEMS Based Piezoelectric System. Proceedings of the 2012 COMSOL Conference in Bangalore.

11. Otterstedt, J-E.; Brandreth, D.A. 1998. Small Particles Technology. Springer, 233.

http://dx.doi.org/10.1007/978-1-4757-6523-6.

V. Ostasevicius, E. Golinka, V. Jurenas, R. Gaidys

HIGH FREQUENCY SEPARATION OF SUSPENDED MICRO/NANOPARTICLES

S u m m a r y

The influence of high frequency vibrations on the large volumes of fluids are not sufficiently studied, but the ultrasound separation of microparticles from liquid in microchannels is known technique. Acoustic standing wave technology creates new opportunities to improve advanced particle and cell separating systems. In this paper an interesting case of using the acoustic pressure method in the vertical plane is considered. The frequencies that were used lie below the threshold of the ultrasonic range and are unique in their structure. The case of interaction of a bimorph plate with a suspension is considered. The influence of the presence of a liquid on the change in the resonant frequencies and the effect of the exfoliation and transport of particles in the suspension are of interest.

Using a high-frequency bimorph piezo-actuator, a stable acoustic effect on the micro/nano suspension and the effect of radiation pressure on particles in the slurry was obtained. Investigating the active part of the actuator, an image with peak points of membrane deformation was obtained, which is confirmed by theoretical calculations. An experimental setup of the micro/nano suspension excitation with piezoelectric actuator has allowed us to determine the lowpressure areas of acoustic waves, which collect micro/nanoparticles. Laboratory studies have confirmed the modeling results and the ability to receive higher volumes than in the case of purified particles in a microchannel.

Keywords: piezoelectric bimorph, holographic interferometry, mathematical model.

Received April 18, 2017

Accepted June 08, 2017 